\title{
Research Article: Inhibition in proper Ser and in PLCy2 synthesis are the main for causing Osteoarthritis, diabetes and C-lymphocytic leukemia diseases
}

Author Name:

Ashraf Marzouk El Tantawi

Biomedical and Molecular Studies

Corresponding Author:

Ashraf Marzouk El Tantawi,

Citation: Ashraf Marzouk El Tantawi, Inhibition in proper Ser and in PLC $\gamma 2$ synthesis are the main for causing Osteoarthritis, diabetes and C-lymphocytic leukemia diseases

\section{Received Date: 23rd Jan 2022}

Published Date: 3rd Feb 2022

Copyrights: Ashraf Marzouk El Tantawi, This is an open access article distributed under the Creative Commons Attribution License, which permits unrestricted use, distribution, and reproduction in any medium, provided the original work is properly cited.
Purpose of study: Understanding that the inhibition or mutation in S6K and in hydroponic acids ( Ser, Tyr, Pro... ), in BTK and in PLC $\gamma 1$ will lead to inhibition in PLC $\gamma 2$ and in Thromboxane-A 2 then will be the main reasons for chronic lymphocytic leukemia "CLL" disease, where proper S6K /BTK and PLC 22 are main regulations for thromboxane-A synthesis and necessary for B-cells maturations and T-cells modulations.

Also, it's important to Understand main factors that cause and link the Osteoarthritis "OA" with diabetes which are the deficiency in Ser (hydrophobic) amino acids and the mutated S6K productions lead to deficiency or inhibition in Ser phosphorylation signaling which normally is the basis of Ser/Thr phosphorylation signalling which necessarily for Akt, for S6K1 synthesis and necessary for RORs and IFNs synthesis, and also necessary for proper PLC $\gamma 2$ productions, where $\mathrm{S} 6 \mathrm{~K}$ is the main regulator for ATPase, for ribosomes, for OPA1 repair, and for proper PLC $\gamma 2$ synthesis, that I have to note that the percentage of the shortages ratio of amino acids or in the increasing in positive linkages are the main ratio that can define the degree and type of specific disease which can differ from other diseases or can linked with the same Syndromes of other diseases. That also the shortage ratio between the beta Cytokines producions and the ratio of sudden high inflammations productions "and the type of its inflammatory molecules" have to be calculated and considered related to the patients ages (whether child, youth or old ages) and the duration of the chronic disease disease, that some can be confused to differentiate between auto-immune disease and regular disease problems diagnosis.

That, There was a case of a child with 9-year-old who had a suspicion of loose of bone maturation and growth and has a sudden infection in the right lung and a lack of breathing with pain. It was found that there was a pulmonary abscesses in right lung and there was a development with the appearance of an air bag or "inflammatory fluid bag" surrounding respiratory cells in right side. The occurrence of sudden inflammations molecules and their growth was rapid enough faster than IFNs productions and faster than PLC $\gamma 2$ productions due to to the age of the child, "Note some her regular treating doctors diagnosed her medical conditions as a type Autoimmune disease and she has weakened immunity due to sudden fast infection related to her young age".

\section{Highlights:}

increasing in PLC $\gamma 1$ with Deficiency in Ser, deficiency in proper S6K, and decreasing in synthase activity with inhibition in PLC $\gamma 2$ will reflect decreasing in anti-inflammatory processes, reflect Osteoarthritis and diabetes syndromes, and also inhibition in PLC $\gamma 2$ will inhibit TXA2 synthesis and can be the main reason for CLL diseases.

proper healthy PLC $\gamma 2$ are so necessary for increasing re-medulate immune efficiencies, and for re-modulate $\operatorname{IgM}$ and $\operatorname{IgD}$ antigen and T-cells functions, and also proper healthy PLC $\gamma 2$ productions (which depend on PLC $\gamma 1$ and BTK Biosynthesis) are so imp for recover osteoporosis and both Osteoarthritis and diabetes.

inhibition in PLC $\gamma 2$ Bio-Synthesis can reflect decreasing or inhibition in Thromboxane-A 1 percentages and can lead to CLL diseases,

Where, CLL characterized by inhibition in BTK which regulate PLC $\gamma 2$ synthesis, inhibition in main antigen synthesis, and then inhibition in the proper normal Thromboxane-A synthesis (which regulated mainly by PLC $\gamma 1$ and by BTK and then linked to IFNs productions and regulated by PLC $\gamma 2$ proper producions). 
Chronic lymphocytic leukemia (CLL) observed during treatment with B-cell receptor inhibitors pathway including inhibitor of Bruton's tyrosine kinase-PLC $\gamma 2$, where, CLL can be strongly linked to Osteoporosis "OA" and Linked to both Osteoarthritis and diabetes too.

\section{Keywords:}

Phospholipase C-1 "PLC $\gamma 1$ ",

Phospholipase C-2 "PLC $\gamma 2$ "necessary for anti-inflammatory steps,

Osteoarthritis OA tissue cells

Osteoporosis tissue cells

Osteoclast processes_Osteoblast processes

Ser/Thr phosphorylation signaling ,

Deficiency in PS/-Thymine-kinases reflect mutated S6K, deficiency in PLC $\gamma 2$, deficiency in B cells and T-cells modulation, and deficiency in OPA1 repair,

S6K, estrogen, androgyne,

JAK state signaling

diabetes pathogenic tissue cells

Tyrosine phosphatase

PTPs

Colony-stimulating Factor-1 "CSF-1"

inositol-1,4,5-triphosphate (IP3) and Diacylglycerol

thromboxane-A "TXA2"

CXCR4, CXCL12

pathogenic chronic lymphocytic leukemia (CLL) tissue cells

B cells and B cells receptors "BCR"

osteprogenitor pathway

Fibroblast growth factor receptor 2 "FGFR2"

interferon regulatory factors (IRFs)

_antigen-specific immunoglobulin $\operatorname{IgM}$ and $\operatorname{IgD}$

\section{Abstract:}

Proper S6K /BTK and PLC 22 synthesis (which regulated by Ser phosphorylation pathway) are main regulations for thromboxane-A "TXA2" synthesis, and necessary for B-cell maturations and T-cells modulations and functions.

The main reasons for causing Osteoarthritis "OA" and diabetes diseases (that are linked together) are the deficiency of Ser amino acids and decreasing of Ser phosphorylation signalling pathway which necessary for proper S6K productions, where normally the Ser phosphorylation signalling pathway is the basis of Ser/ Thr phosphorylation signalling and is necessary for proper Akt, S6K1 synthesis and necessary for RORs and IFNs synthesis and also necessary for running proper BTK for PLC $\gamma 2$ productions, where $\mathrm{S} 6 \mathrm{~K}$ is main regulator for ATPase and for proper PLC $\gamma 1$ and for PLC $\gamma 2$ synthesis which necessary for bone growth and for modulating immune efficiency.

Osteoarthritis "OA" is characterized by a sharp expression in Gamma-Phospholipase C-1 "PLC $\gamma 1$ ", with decreasing "or inhibition" in PLC $\gamma 2$ "PLC beta" productions due to inhibition or mutation in S6K and then in BTK.

The increasing in PLC $\gamma 1$ with Deficiency in Ser amino acids will lead to deficiency in Ser phosphorylation signalling, and decreasing in synthase activity that will reflect down regulations in BTK pathways and lead to inhibition in PLC $\gamma 2$ productions which will reflect diabetes (inhibition in Estrogen with the production of Androgen instead of estrogen) and can reflect Osteoarthritis"OA" prognosis depend on the percentage of Deficiency or inhibition in basic amino acids and its basic necessary signaling pathways.

The proper S6K are so necessary for reactivating both PLC $\gamma 1 \& 2$
, where phospholipase $\mathrm{C} \gamma 2$ (PLC $\gamma 2)$ is activated from a variety of cell surface receptors such as SyK "S6K".

The B-cells are promoted by the function and activities of both PLC $\gamma 1 \& 2$, but the deficiency in Ser amino acids will reflect decreasing in Ser phosphorylation pathways and then decreasing in Estrogen synthesis, with increasing in Androgen synthesis which lead to decreasing in PLCs isoforms production and lead to pathogenic diabetes problem. So T2DM is strongly connected with OA diseases are linked together that deficiency in Ser amino acids and their phosphorylation can cause both diseases and any early step from any of those two or more similar diseases can lead to the other.

Pathogenic type 2 diabetes associated with progressive betacell impairment due to the mutations in the production of S6K1 and inhibition in the PLC $\gamma 2$ which due to inhibition or decreasing in Ser phosphorylation signaling during mTOR Ser/ Thr phosphorylation pathways that can reflect Inhibition in The releasing of PS/T-Thymine Kinases and PS/T-Cytosine kinases chains (mTORC1) which are depending on availability of hydrophobic amino acids synthesis including Ser and Tyr which can be modified by synthetase enzymes for creating active gamma-subunits (upon synthetase effects) that can be modified by synthase effect for Beta-subunit synthesis "PLC $\gamma 2$ " then will be modified by phospholipase effects for alpha subunits productions.

The releasing of PS/T-Thymine -Kinase and PS/T-Cytosine -kinase chains (mTORC1) from the phosphorylations of Ser amino acids is so necessary steps for normal proper S6K productions, which necessary for IFN-Gamma and for PLC $\gamma 1$ productions, and therefore necessary for normal PLC $\gamma 2$ synthesis upon "BTK activity" which is necessary for B-cell maturations, for T-cells modulations, for modulating anti-inflammatory steps and procedures, for thromboxane-A synthesis, and for bone growth and modulation.

Inhibition in PS/T-Thymine -Kinase and PS/T-Cytosine -kinase chains (mTORC1) productions will be the main reason for inhibition the beta subunits productions that can be the reason of decreasing in the hyperpolarization and then electrical activity will lead to decreasing in the abolition of Ça+ .

Deficiency in conversion of glutarate to glutamate and decreasing in proline biosynthesis can affect on cartilage synthesis and bone growth due to decreasing in stimulating mitochondrial OPA1 oxidations.

It's imp to note that Tyrosine phosphatase

PTPs are important regulator of chondrogenic patterning and are critical regulators of tyrosine phosphorylation that it's activity depends on Tyr, Ser synthesis (hydrophobic acids) and on JAK state signaling activities.

And so, the proline-rich tyrosine kinases regulate proper PLCs isoforms which compete for binding site at the very $\mathrm{C}$ terminus of fibroblast growth factor for osteorogenitor embryonic development, and bone formations.

Synthetase is the main regulator for PLC $\gamma 1$ activities followed by synthase effects which is the main regulator for betasubunits "PLC $\gamma 2$ " productions which is able to "upregulate phospholipase abtivity" for alpha subunits (PLC-alpha) productions for reactivating fibroblast growth factor receptor (FGFR2), for reactivating both $\operatorname{IgM}$ and $\operatorname{IgD}$ and for TLR4 productions for osteoblast processes .

Where, PLC $\gamma 1$ competes for a binding site at the very $\mathrm{C}$ terminus of FGFR2 for embryonic development and bones growth, where, PLCs isoforms are involved in multiple stages in TLR4, interferon, and in anti-inflammatory steps . 
And also , PLC $\gamma 1$ recruit to CSF-1 is following imp stages for producing PLC $\gamma 2$ which is necessary for activating antiinflammatory where, IFN- $\gamma$ activates PLC- $\gamma 2$ via an upstream of tyrosine kinase.

PLC $\gamma 1$ recruited to CSF-1 for two pathways activities 1st / re-activating IFNs productions which regulate MHC class 1 and class two for modulating cell-surface protein activities, 2nd / activating PLC $\gamma 2$ for modulating T-cells, where PLC $\gamma 1$ involved in the production of TRIM22 for mediating antiviral activities and anti-inflammatory processes through reactivating IFNs productions for PLC $\gamma 2$ synthesis.

PLC $\gamma 2$ are so imp in anti-inflammatory processes and can be considered as having the main roles for thromboxane-A synthesis.

Inhibitions or mutations in S6K, in BTK and then in PLC 22 productions will cause an inherent or inhibition in CXCL12 then followed by inherent or inhibition in CXCR4 then reflect inherent or inhibition in the regulation of B-cell growth through mutations in IgM and in $\mathrm{IgD}$.

Proline amino acids is necessary for reactivate OPA1 anabolic oxidations (started by synthetase, then synthase, then phospholipase for producing gamma "PLC $\gamma 1$ ", then beta "PLC $\gamma 2$ ", and then alpha "PLC-a" subunits respectively ) for cartilage synthesis which promote PLC $\gamma 2$ synthesis necessary for bone growth including antigen and thromboxane-A synthesis.

\section{Introduction :}

Osteoarthritis is characterized by a sharp expression in GammaPhospholipase C-1 "PLC $\gamma 1$ ", with decreasing in PLC $\gamma 2$ "PLC beta" which improved by phospholipase oxidations for producing PLC alpha which necessary for proliferations and calcium entry ", where PLC $\gamma 1$ was highly expressed in human OA chondrocytes [1] ) which is implicated processes including mitogenesis and calcium entry.

Phospholipase $\mathrm{C}$ isoforms (PLCs) are essential mediators for cellular signaling and for cellular metabolism .

PLCs regulates multiple cellular processes including proliferations and biological bones growth by generating bioactive molecules such as inositol-1,4,5-triphosphate (IP3) and diacylglycerol.

That, PLC $\gamma 1$ basis of inhibition-driven autophagy of IL$1 \beta$-treated chondrocyte confers cartilage protection against osteoarthritis. [2] the only presence PLC $\gamma 1$ has the roles of analyzing biological molecules "Osteoclast" through expressing its own specified functions, while PLC $\gamma 2$ has the role of functioning PLC $\gamma 1$ for running anti-inflammatory processes and for promoting proliferation through activating phospholipase for activating PLC alpha for running proliferation.

So slightly inhibition or decreasing in PLC $\gamma 1$ will decrease osteoclast and also re-functioning PLC $\gamma 1$ for reactivating the expression of PLC $\gamma 2$ "which reduce the analyzing function of PLC $\gamma 1$ ", then will give the priority for PLC-beta "PLC $\gamma 2$ " for production by beta-oxidations for activating anti-inflammatory processes, and for promoting PLC-alpha production which necessary for proliferations functions that will activate osteoblast processes, bone growth, and cells proliferation "modulations". The availability of Proline amino acids are necessary for activating and accelerating OPA1 oxidative processes which activate cartilages synthesis through PLC $\gamma 2$ synthesis, and the necessary of hydrophobic amino acids availability and proper synthesis in vivo is important for gamma subunits synthesis regulated by synthetase enzymes "eg :Tyr, Leu, Pro, Gly, Ser, ... etc". Proline with hydroponic acids can activate and accelerate proper OPA1 oxidative processes which promote and activate necessary anabolic cycles by activating BTK which regulate PLC $\gamma 2$ synthesis and bone growth and for modulating immune effectiveness.

The Deficiency in the conversion of glutarate to glutamate and decreasing in proline biosynthesis strongly affect on cartilage synthesis due to decreasing in the activation of mitochondrial OPA1 oxidative processes. [3] Also, deficiency in the mitochondrial OPA1 membrane repairs process of can reflect deficiency in the proper S6K productions (which necessary for ATP and GTPase synthesis where GTPase is strong regulator factor for OPA1 repair ) that will lead to deficiency in OPA1 mitochondrial function that will lead to decreasing in PLCs synthesis (decreasing in PLC $\gamma 2$ ) then in SIRP $\alpha 1$, and in TLR4 biosynthesis, that can reflect increasing in catabolic analyzing processes (due to increasing in PSTG-kinases and PSTA-kinases "for a limit" with decreasing in PS /T Thymine kinases and in PS/T-Cytosine kinases productions which will lead to diabetes, where in diabetes the synthetase activities can be increased that can analyze phospholipids, foreign molecules and Enterstatium fluid lead to decreasing in the synthesis of anti-inflammatory tools eg PLC $\gamma 2$. Proper S6K1 synthesis can promote ATPase and GTPase productions through OPA1 repair for activating RORs pathways, for activating BTK pathways and for PLC $\gamma 2$ productions, where all are depending on the four types of kinases production from Ser Thr phosphorylation pathways which activate proper PLCs productions, proper IFNs activation, proper MHCs synthesis and proper bone growth synthesis with beta-cells proper maturations and T-cells modulations.

\section{Method and results:}

Proper S6K /BTK are regulating PLC $\gamma 2$ synthesis and are regulating proper thromboxane-A synthesis, B-cell maturations and T-cells modulations .

Where, it's so important to Understand main factors that cause Osteoarthritis "OA" and diabetes which are the deficiency of Ser amino acids which lead to mutated S6K production due to deficiency or inhibition in Ser phosphorylation signaling which normally is the basis of Ser / Thr phosphorylation signalling that are necessary for proper Akt, S6K1 synthesis and necessary for RORs and IFNs synthesis and also necessary for proper PLC $\gamma 2$ productions.

Proper S6K productions through availability of Ser are main regulator for ATPase synthesis and GTPase which necessary for OPA1 repair, and for BTK pathways and proper PLC $\gamma 1$ synthesis which regulate PLC $\gamma 2$ synthesis for necessary bone growth and cartilage synthesis.

Osteoarthritis "OA" is characterized by a sharp expression in Gamma-Phospholipase C-1 "PLC $\gamma 1$ ", with decreasing "or inhibition" in BTK which lead to decreasing in PLC $\gamma 2$ "PLC beta" that will reflect decreasing in beta-cells maturation and functions and in T-cells modulations.

The increasing in PLC $\gamma 1$ with Deficiency in Ser will lead to mutated $\mathrm{S} 6 \mathrm{~K}$ production, and decreasing in proper synthase activity and decreasing in BTK processes that will lead to inhibition in PLC $\gamma 2$ synthesis that will reflect Inhibition in Estrogen synthesis and increasing Androgyne synthesis that will give the Symptoms of diabetes and Osteoarthritis "OA" diseases.

we'll discuss why both diseases are connected and their causes depend mainly on availability of Ser and hydroponic amino acids, and dépend on the proper S6K synthesis then on the Tyr and other hydroponic acids synthesis and their phosphorylation 
signaling pathway and JAK for the Synthesis of their Receptors. Deficiency in the proper S6K, in Ser and in Tyrosine synthesis "regulated by synthetase" will lead to increasing in PLC $\gamma 1$ with decreasing in PLC $\gamma 2$ synthesis (which are Regulated by availability of PS /T-Cytosine k and PS /T-Thymine Kinases) will lead to androgen synthesis instead of estrogen "diabetes" and Osteoarthritis "OA" diseases :

PLC $\gamma 1$ is a protein molecules that it's activity depending on Tyr phosphatase and gamma common receptors synthesis which regulated by JAK STAT signaling, and also regulated by synthetase enzyme where synthetase is the main second enzyme in OPA1 chains after COX enzyme (followed by synthase and phospholipase respectively ) and necessary for hydroponic acids synthesis for gamma active subunits synthesis (or extraction) , that synthetase enzymes is so necessary for creating signals transmission which can reactivate mTOR Ser/Thr signaling pathway and for re-producing the active gamma-subunits which upon JAK signaling will produce their active receptors necessary for activating gamma subunits "PLC $\gamma 1$ " beta-subunits "PLC $\gamma 2$ " synthesis which upon phospholipase will produce alpha subunits "PLC-alpha" for activating proliferations, and bones growth. The PLC $\gamma 1 / \mathrm{PLC} \gamma 2$ double-deficient B cell progenitors have reduced expression of genes related to $\mathrm{B}$ cell lineage, IL-7 signaling, and cell cycle. [4] That the activities of both PLC $\gamma 1 \& 2$ are linked to each other and are so necessary for re-activating B-cells maturation, where, PLC $\gamma 2$ regulate the productions antigen-specific immunoglobulin necessary IgM and $\operatorname{IgD}$ synthesis necessary for anti-inflammatory processes, and necessary for T-cells modulations, therefore the deficiency or mutations in PLC $\gamma 1 \& 2$ will lead to decreasing in or lead to Malignant transformation in B cells that can cause mutations or inhibition in $\operatorname{IgM}$ and in $\operatorname{IgD}$ synthesis and will lead to inhibition or mutations in TXA2 synthesis too that will lead to a cancer problem as chronic lymphocytic leukemia (CLL) and can cause several other pathogenic problems as diabetes and OA diseases. B-cells are promoted by the productions of both PLC $\gamma 1$ which upon BTK will regulate PLC $\gamma 2$ synthesis, where PLC $\gamma 1$ synthesis mainly depends on mTOR Ser/Thr phosphorylations signalling pathways (mTS/TP) and dépend on proper S6K synthesis "that deficiency in Ser amino acids will reflect decreasing in the productions of the two types kinases PSTT-K and PSTC-k that will lead to mutations in S6K synthesis and lead to decreasing in Estrogen synthesis with increasing in Androgen synthesis which lead to pathogenic diabetes diseases.[5]

Proper S6K synthesis is depending on availability of Ser amino acids and on the production of the two kinases PSTTK and PSTCk that are so necessary for reactivating ribosomal ATPase which is necessary for repairing the mitochondrial OPA1 membrain (through regulating GTPase productions), where proper OPA1 is necessary for activates and regulating proper PLC $\gamma 1$ productions and for "PLC $\gamma 2$ " synthesis upon synthase effect for $\mathrm{B}$-cell receptor synthesis for B-cells maturation, and for antiinflammation, then followed by creating PLC-alpha synthesis upon phospholipase functions for promoting proliferations and bone growth through SIRPa and TLR4 productions.

In case of deficincy in mTOR Ser/Thr phosphorylations signalling due to deficiency in Ser phosphorylation will produce non proper mutated S6K "missing Ser hydrophobic amino acids" that will lead to diabetes pathogenic problems, and will lead to inhibition in PLC $\gamma 2$ or will lead to mutated PLC $\gamma 2$ in some cases depending on the percentage of Deficiency of necessary hydroponic (Tyr, leu, Pro,... etc) that will lead to inhibition in
Estrogen which is the substrate for RORs pathways and will lead to increasing in Androgyne instead of Estrogen that will inhibit PLC $\gamma 2$ synthesis and will lead to diabetes, and OA diseases and can lead to cancer pathogenesis in the inhibition or mutation in TXA2 synthesis.

Pathogenic type 2 diabetes associated with progressive beta-cell impairment due to the mutations in the production of normal Estrogen (that Androgyne is formed instead of Estrogen) due to missing of Ser phosphorylation signaling during mTOR Ser/ Thr phosphorylation pathways due to Inhibition or decreasing in the releasing PS/T-Thymine -Kinases and PS/T-Cytosine -kinases (mTORC1) where those two kinases synthesis depending on the availability of Ser amino acids and depend on Ser phosphorylation pathway, (where synthetase enzymes regulate hydrophobic amino acids synthesis $8 \mathrm{n}$ vivo) where through gamma oxidations by synthetase will promote gammasubunits that will be modified by synthase effect to produce active Beta-subunits synthesis which are necessary for "antiinflammations and for promote alpha subunits synthesis upon phospholipase effects "alpha-oxidations" which necessary for proliferation respectively.

The releasing of PS/T-Thymine -Kinase and PS/T-Cytosine -kinase chains or (mTORC1) from specifically the phosphorylations of Ser signalling pathway is so necessary for the mechanism of normal and proper S6K productions which necessary for ATPase synthesis, for IFN-Gamma productions, and for activating BTK which necessary for promoting PLC $\gamma 2$ productions which is necessary for B-cell maturations and functions, for T-cells modulations, for modulating antiinflammatory steps and processes, for thromboxane-A synthesis, and for bone growth and maturation.[6A*]

The inhibition in PS/T-Thymine-Kinase and PS/T-Cytosine -kinase (mTORC1) productions will be the main reason for the inhibition of the beta subunits productions "PLC $\gamma 2$ " that can be the reason of decreasing in the hyperpolarization and then electrical activity will lead to decreasing in the abolition of $\mathrm{C} a+$ which will lead to decreasing in blood pressure and $\mathrm{Ça}$ precipitations in blood vessels.

Also, the deficiency in Tyrosine amino acids will prevent the production of tyrosine phosphatase which needed for the synthesis of phospholipase C $1 \& 2$ that promote cellular proliferation, and the reduction and deficiency in Tyr amino acids "hydrophobic acids" will reduce or inhibit Drutons tyrosine kinases "DTK".

Now it is important to consider that proper $\mathrm{S} 6 \mathrm{~K}$ is the main regulator for PLCs isoforms synthesis which depend on S6K productions, and it has been reported that the phospholipase $\mathrm{C} \gamma 2$ (PLC $\gamma 2$ ) is activated from a variety of cell surface receptors such as SyK "S6K", and BTK which phosphorylate and activate PLC $\gamma 2[6]$.

Proper S6K1 synthesis is the basis for ATPase, and GTPase synthesis and also is the basis for ribosome repair where, GTPase is necessary for G-protein synthesis, for OPA1 membrane repair, and for ribosomal repairs that always necessary for regulating cellular growth and anti-inflammatory Processss.

As the GTPase is a regulator tool for $\mathrm{BH} 4$ and NO 3 productions for synthase repair and activity,

As, S6K1 is the main regulator for PLC $\gamma 1$ synthesis and then for PLC $\gamma 2$ synthesis upon synthase functions which later will migrate for beta-cells maturation and survival upon productions of firstly CXCL12 then CXCR4 productions .

Also, it has been approved that T2DM is connected with OA and both are having the same reason of causing their pathogenic 
disease, where T2DM has a pathogenic effect on OA through 2 major pathways involving oxidative stress and low-grade chronic inflammation resulting from chronic hyperglycemia and insulin resistance [7]. Pathogenic type 2 diabetes associated with progressive beta-cell impairment due to the not normal production of insulin which due to deficiency of Ser phosphorylation pathway during mTOR Ser/Thr phosphorylation pathways that will not produce normal S6K "due to deficiency in Ser and some other necessary amino acids (mainly Ser, Tyr, Leu, Pro a.a.) then will lead to decreasing "or mutation" in the S6K productions, that will lead to Androgen production instead of Estrogen where Estrogen characterized by presence of Ser in their molecules "and is the substrates for ROR anabolic signaling pathways", that will lead to high ATPase productions (due to availability of purines with decreasing in pyrimidine synthesis) with deficiency estrogen synthesis that later will promote the IFN gamma, IFN-beta, and alpha that can lead to increasing in "catabolic processes" with decreasing in the ROR pathways "anabolic process" and decreasing in proper PLC $\gamma 2$ productions that will reflect $\mathrm{Ca}+$ precipitations and arterial hypertension.

Where, it has been reported that insulin activates the K-ATP channels of pancreatic $\beta$-cells and islets, resulting in membrane hyperpolarization, and the abolition of [Ca2+]i oscillations [8]. And, the low abolition of $[\mathrm{Ca} 2+]$ i oscillations in the case of T2DM indicates decreasing or inhibition in pyrimidine synthesis "regulated by synthetase", decreasing in synthase functions, and decreasing in PLC $\gamma 2$ synthesis "that has the role of modulating inositol 1,4,5-trisphosphate-mediated calcium oscillations for bone growth ". Also, decreasing in membrane hyperpolarization can give reflection of decreasing in OPA1 synthase oxidations which reflect decreasing in membrane hyperpolarization and decreasing in PLC $\gamma 2$ synthesis.

(PLC $\gamma 1)$ can be reactivated by platelet-derived growth factor "GF" receptors, insulin-like GF 1 receptor (which reflect deficiency in proper cells and bones growth), but in brief PLC $\gamma 1$ productions can produced and re-functioned by several active growth factor (GF) receptors through feedback and by firstly reactivating synthetase followed by synthase then phospholipase which promote growth factor activities as epidermal GF receptor [EGFR], and platelet-derived GF receptor, where due to activating GFs processes it will be responsible for increasing hyperpolarization and functioning CA throughout the synthesis of PLCs that will responsible for running the pathway of bone growth and cellular biosynthesis processes.

The main PLC $\gamma 1$ proper activities is regulated firstly by main ribosomes and by proper S6K productions from mTOR Ser / Thr phosphorylation pathways followed by JAK STAT signaling for producing the Tyr-phosphatase, gamma common receptors, and other necessary helical proteins receptors which adopt and activate PLC $\gamma 1 \& 2$ synthesis and activities for anti-inflammatory, for B-cells maturation, for T-cells modulation, and for bone growth and proper cellular proliferation.

PLC $\gamma 1$ is a necessary Protein regulated firstly by chromosomes, then by ribosomes activities and by S6K which produced from mTOR Ser /Thr signaling pathway that regulated firstly by OPA1 synthetase and then activated by JAK STAT signaling for creating necessary receptors for both PLC $\gamma 1$ and then PLC $\gamma 2$ productions, where PLC $\gamma 2$ is also regulated by BTK for proper PLCs isoforms productions for cellular proliferation and bones growth.

Hydrophobic acids such as Tyrosine, Ser, proline are necessary for facilitate the cellular and B-cells maturation and survival that protect proliferation processes of bones development (also can activate tumor growth in case of synthase dysfunction when lose or deprived of some necessary amino acids) through facilitating OPA1 oxidative functions (that proline is necessary for OPA1 enzymes activities which activate their function and bone cartilage growth ) rand activate BTK pathways which necessary for FGFR2 gene expression for bones developments. Where, Tyrosine amino acids increase alertness and bone development through activating tyrosine kinases, that Tyrosine phosphatases which are potential therapeutic targets for fighting bone disorders [9].

Protein tyrosine phosphatase (PTP) gamma (carry-ve charge regulated firstly by synthetase gamma-oxidations) has been proposed to be an important regulator of chondrogenic patterning, where PTPs are critical regulators of tyrosine phosphorylation at multiple stages of bone development and metabolism [10]. And, proline-rich tyrosine kinases regulate osteprogenitor cells and bone formations, [11] so Tyrosine and Proline (where their synthesis firstly regulated by synthetase in vivo) are regulated by PIPs and are critical regulators for multiple stages in bone development started by cartilage synthesis.

Tyrosine, Ser and proline are essential hydrophobic acids that produced in vivo upon the effects of synthetase enzymes on nutrients-mTOR, and on inflammations molecules for running pyrimidine synthesis for creating and improving (modulating) active Gamma-subunits for PLC $\gamma 1$ synthesis which modulated and regulate the beta subunits "PLC $\gamma 2$ " (upon BTK regulation) which necessary for increasing and modulating anti-inflammatory efficiency, then the PLC $\gamma 2$ will be modulated for producing alpha "PLC-alpha" active subunits productions which necessary for proliferation, B-cells maturations, and bone growth.

Gamma-subunits firstly moderated by JAK STAT signaling for producing their own active gamma subunits receptors (as Gamma-common and other helical proteins) which can be promoted by IFN gamma too for re-activating PLC $\gamma 1$, PD1, MHC-class- 1 and class two, (where PLC $\gamma 2$ promote antigens $\operatorname{IgM}$ and $\operatorname{IgD}$ ), then $\mathrm{MHC}$ class two promote the SIRP $\alpha 1$, TLR4, and PD-L1 productions necessary for bone growth, cells developments and T-cells modulations.

PLC $\gamma 1$ competes for binding site at very $\mathrm{C}$ terminus of FGFR2 for embryonic development and bones growth, where, PLC isoforms are involved in multiple stages in TLR4, and in interferons production:

PLC $\gamma 1$ competes for a binding site at $\mathrm{C}$ terminus of fibroblast growth factor receptor (FGFR2) (which plays an important role in bone growth, particularly during "embryonic development" and is sufficient to upregulate phospholipase activity [12]. That, S6K and synthetase regulate PLC $\gamma 1$ production followed by BTK and synthase effects for beta-subunits ( "PLC $\gamma 2$ ") productions which stimulate phospholipase "abtivity" for up regulating phospholipase activity for active alpha subunits (PLC-alpha) productions which can reactivate the production of fibroblast growth factor and their receptors (FGFR2) for full proliferations cycles, bone growth, cells maturation and T-cells modulations.

Their are strong relationships between PLC $\gamma 1 \& 2$ bio-activities and productions of the MHC class 1 and two which promote SIRP $\alpha 1$, TLR4, and PDL1 productions which are necessary for proliferation, cells modulations and T-cells modulations.

Only Synthetase enzyme in OPA1 mitochondrial membrane are having the ability of hydrolysis biological molecules, inflammations and phospholipid membranes in vivo ,but 
normally followed by the effects of synthase for moderate gamma subunits for producing PLC $\gamma 2$ which will be moderated by phospholipase effects for PLC alpha production, but in deficiency in the synthase activities or in presence of mutated S6K the Osteoclast will be activated, where Osteoblast activity is characterized by proper availabilities of $\mathrm{S} 6 \mathrm{~K}$, synthase activities, and PLC $\gamma 2$ synthesis.

Some PLCs isoforms synthesis are involved in multiple stages in TLR4 and interferons regulatory factors (IRFs) synthesis [13]. Where it means the involvement of only PLC $\gamma 2$ in TLR4 synthesis and in promoting IFN-beta productions for modulate anti-inflammatory effectiveness, but PLC $\gamma 1$ is promoting IFN gamma activities (PLC $\gamma 1<\neg \neg>$ IFN gamma) which responsible for promoting MHCs class-1 and class two then SIRP $\alpha$ 1, TLR4 and PDL1 productions for proliferation, bone growth and $\mathrm{T}_{-}$ cells modulations. Also the availability of proper S6K1 for PLC $\gamma 1$ are so necessary for activating IFN-beta and for TLR4. So, proper PLC $\gamma 1$ can be considered as important tools produced in vivo for activating IFN gamma and vice versa necessary for regulating PLC $\gamma 2$ upon BTK activity for antiinflammatory processes which will be upgraded and moderated by phospholipase activities for PLC alpha, SIRP $\alpha 1$ TLR4 and and for PD-L1 productions.

Therefore, PLC $\gamma 1$ regulate PLC $\gamma 2$ production which regulated by tyrosine phosphatase receptors and by phospho-tyrosine receptors "PTyr-R"

for activating PLC $\gamma 2$ productions which then regulate PLCalpha reproduction for bone growth, for B cells maturation, and for promoting anti-inflammatory steps.

Where, PLC $\gamma 2$ are basically depend on JAK signaling for SH2B adaptor protein "which are a Tyr kinase receptor family" that necessary for BCR mediate B cells maturations [14"] phosphotyrosine "PTyr" are necessary for PLCs synthesis, and for SHP1Src homology region 2 domain containing phosphatase 1 for regulating PLCs productions, for stimulate IFNs productions for anti-inflammatory processes and for proliferations, B-cells maturation, and T-cells modulations.

PLC $\gamma 1$ is associated with numerous inflammatory diseases due to deficiency in synthase (which depend on availability of Ser and Tyr ) for PLC $\gamma 2$ productions, that in some diseases the mutation in S6K can be the main for causing those diseases (due to the deficiency in Ser phosphorylation signaling) and in other cases due to deficiency in proline and in Tyrosine hydroponic amino acids, that healthy immune is depending on the productions of PLC $\gamma 1$ is for acting firstly on infections (by Gamma oxidation) which will promote PLC $\gamma 2$ productions upon the synthase and BTK activities for modulating anti-inflammatory processes, and then will promote proliferations upon PLC alpha productions due to phospholipase regulations.

PLC $\gamma 1$ recruit to Colony-stimulating factor-1 "CSF-1" is followed by imp stages for producing PLC $\gamma 2$ which is necessary for activating anti-inflammatory cycles through activating IFNs which re-activate PLC- $\gamma 2$ via upstream of tyrosine kinase :

The PLC $\gamma 1$ has the specificity toward colony-stimulating factor receptor synthesis (CSF-1) signaling which expressed on the cell surface that can cause the cells to proliferate and differentiate into specific blood cells, and considered as a class III receptor tyrosine kinase that associated with Neuroinflammation, where $\mathrm{PLC} \gamma 1$ is recruited to the CSF-1 receptor following exposure to the cytokine. [15] PLC $\gamma 1$ specify for recruit to CSF-1 which necessary for promoting PLC $\gamma 2$ synthesis for firstly re-activating anti-inflammatory steps then followed promoting proliferation steps through activating PLC alpha, SIRP $\alpha 1$, TLR4 and then PD-L1 productions.

CSF-1 is a members of the IL-1 receptor family regulated by Gamma oxidation by PLC $\gamma 1$ for promoting PLC $\gamma 2$ synthesis for re-stimulations IFN beta productions for modulating antiinflammatory cycles and efficiency .

That, CSF1R-expressing cells may play an anti-inflammatory role or a cancer-suppressive role. [16] As PLC $\gamma 1$ recruiting to CSF-1 for regulating PLC $\gamma 2$ synthesis so CSF-1 play necessary role in promoting anti-inflammatory processes which regulated firstly by mitochondrial OPA1 enzymes, by proper S6K production, and by PLC $\gamma 1$ synthesis .

Also , Tripartite motif (TRIM) 22 plays an important role in interferons (IFNs)-mediated antiviral activity and the Induction of TRIM22 by IFN- $\gamma$ Involves JAK and PC-PLC/PKC. [17] So PLCs synthesis modulate and regulate Tripartite motif (TRIM) 22 too (which has antimicrobial activities) productions through activating IFNs production.

Also, IFN- $\gamma$ activates PLC- $\gamma 2$ via an upstream tyrosine kinase to induce activation of PKC- $\alpha$.

[18] that PLC $\gamma 2$ regulated by PLC $\gamma 1$ which can promote IFNgamma production (through feedback) which has a variety of activities including PLC $\gamma 2$ re-productions upon the necessity regulations of the upstream of tyrosine kinases for re-activating PKC- $\alpha$.

PLC $\gamma 1$ recruited to CSF-1 for two pathways activities 1st / re-activating IFNs productions which regulate MHC class 1 and class two for modulating cell-surface protein activities, 2nd / activating PLC $\gamma 2$ for modulating T-cells, where PLC $\gamma 1$

involved in the production of TRIM22 for mediating antiviral activities and anti-inflammatory processes through reactivating IFNs productions for PLC $\gamma 2$ synthesis which modulate T-cells and activate bone growth with activating necessary proliferation. And also PLC $\gamma 1$ promote IFN gamma which regulate MHCclass-I, MHC class-2 synthesis which promote, SIRP $\alpha 1$, TLR4, and PD-L1 synthesis .

Note that the inhibitions of PLC $\gamma 2$ productions with PLC $\gamma 1$ productions will lead to Osteoclast, but the proper balance of both PLC $\gamma 1$ and PLC $\gamma 2$ productions will lead to osteoblast where PLC $\gamma 2$ are connected to IFNs productions too.

Also, the Colony-stimulating factor-1 "CSF-1" requires PI3kinase-mediated metabolism for proliferation [19]

PLC $\gamma 1$ recruited to Colony-stimulating Factor 1 "CSF-1"

Depending on mTOR-Ser/Thr phosphorylation signaling for p13k and for proper S6K productions.

And, The inhibitions of of fatty acid synthase "FAS" activity by C75 is resulted in down regulation of phospho-AKT. [20]

The inhibition in synthase will reflect down regulations in OPA1 membrane and therefore Down regulation in p13k Akt and in S6K productions which necessary for ribosomes repair and for OPA1 repair upon GTPase re-synthsis.

PLC $\gamma 2$ synthesis activate Osteoblast but PLC $\gamma 1$ production with inhibition in PLC $\gamma 2$ will activate Osteoclast (OC) by inhibiting the inositol 1,4,5-trisphosphate- PLC $\gamma 1 \& 2$ synthesis are remodulating variety of cellular pathways including osteoclast (OC) differentiation.

Where, PLC $\gamma 2$ production is important to be in proper balance with PLC $\gamma 1$ synthesis for running osteoblast and for inhibiting osteoclast, where the increasing in PLC $\gamma 1$ productions with inhibition in PLC $\gamma 2$ will activate osteoclast (OC) by inhibiting re-modulating inositol 1,4,5-trisphosphate "which mediated calcium oscillations and the up-regulation of the nuclear 
transcription factor NFATc1". [21]

That, inositol 1,4,5-trisphosphate and diacylglycerol productions require phosphoinositide synthase (PIS) for modulating OC differentiation through regulating transient receptor potential (TRP) channels which requires hydrolysis of phosphatidylinositol 4,5-bisphosphate (PIP)

resulting in the generation of inositol 1,4,5-trisphosphate (IP3) and diacylglycerol (DAG).

OPA1 synthase is necessary for creating sphosphoinositide synthase (PIS) "regulated by proper S6K production which needed for GTPase synthesis which necessary for OPA1 membrane repairs".

Both PLC $\gamma 1$ and sphosphoinositide synthase (PIS) are imp for promoting PLC $\gamma 2$ productions which necessary for upregulate phospholipase activity for PLC alpha for proliferations and bone growth, Where, increasing in PLC $\gamma 1$ "with reduction or inhibitions in PLC $\gamma 2$ productions will activate osteoclast but the reactivating proper PLC $\gamma 2$ synthesis will activate Osteoblast. PLC $\gamma 2$, independent of PLC $\gamma 1$, was required for receptor activator of $\mathrm{NF}-\mathrm{\kappa B}$ ligand-induced osteoclastogenesis by differentially regulating nuclear factor of activated $\mathrm{T}$ cells c1 (NFATc1), [22] proper PLC $\gamma 2$ Pathway for modulating osteoclastogenesis

Processes mediated by modulating T-cells To complete first the construction of anti-inflammations and the protection followed by the process of building bones growth and cells proliferation in the safety and protection of T-cells and macrophages.

$\mathrm{BTK}$ regulate PLC $\gamma 2$ synthesis which regulate both BCR and Thromboxane-A 2 synthesis, where, CLL disease due to full inhibition in PLC $\gamma 2$ :

Phospholipase $\mathrm{C} \gamma 2$ is Critical for Dectin- 1 mediated Ca2+ Flux and Cytokine Production in Dendritic Cells [23].

PLC $\gamma 2$ has a critical activity in dendritic cells, where is having a Critical function for Development of a Murine Model of Inflammatory Arthritis. [24]

And, as PLC $\gamma 2$ has a critical activity in dendritic cells for activating NF- $\mathrm{\kappa B}$ ligand-induced osteoclastogenesis

by differentially regulating nuclear factor-activated $\mathrm{T}$ cells $\mathrm{c} 1$ "NFATc1"

As PLC 22 production modulate first the capacity of T-cells of dendritic cells .

PLC $\gamma 2$ is critical for B-cell receptor (BCR) for B cells maturation and functions, and PLC $\gamma 2$ participates in TCR signal transduction and plays a role in T-cell selection [25]

It has been reported that Properdin and factor $\mathrm{H}$ production by human dendritic cells modulates their T cell stimulatory. [26]

Properdin is plasma glycoprotein that when activated by PLC $\gamma 1$ (and synthetase) that will be modulated by change unnecessary purines to pyrimidines for rebuilding necessary Tyr, Ser, Pro, then will be directed to $\mathrm{x}$ chromosome for translations and purification for being build by identical necessary sequences for being contain identical six thrombospondin that will be ready to be regulated and modulated by PLC $\gamma 2$ for TXA 2 synthesis and for modulating T-cells which mediate cellular and bone growth. The increasing in PLC $\gamma 1$ productions with deficiency or mutation in S6K and thus in Properdin will inhibit PLC 22 functions and will reflect decreasing in $\mathrm{B}$ cells maturation with decreasing or mutations in the thrombospondin lead to inhibition in TXA2 synthesis and can lead to Autoinflammation and immune dysregulation (APLAID) which can cause rare monogenic autoinflammatory disease.

That, The diverse pathologies associated with PLC $\gamma 2$ are exemplified by distinct genetic variants, where inherited mutations at this locus cause PLC $\gamma 2$-associated antibody deficiency and immune dysregulation. [27]

Thrombine activation is highly reactivate intermediate the true fibrin monomer and it rapidly, and irreversibly. [28]

That Thrombine is activated by PLC $\gamma 2$ which intermediate fibrin monomer.

Where, PLC $\gamma 2$ involved with fibrin formation, where Bruton tyrosine kinase (Btk) activates PLC $\gamma 2$, 11,12 leading to thromboxane A2 (TXA2) synthesis. [29]

So, proper PLC $\gamma 2$ synthesis depend on PLC $\gamma 1$ and on BTK activities that are necessary for regulating thromboxane-A 2 and fibrin and for re-modulating immune and $\mathrm{T}$ cells activities.

Also, the antiplatelet and anti-thrombotic effects of Fc are carried out through oppression of PLC $\gamma 2$ and subsequent DAGPKC-TXA2 and IP3-[Ca2+]. [30]

The activation of PLC $\beta$ through Gq, which results in the formation of IP3 and diacyl glycerol, plays an important role in mediating $\alpha \operatorname{IIb} \beta 3$ activation. [31]

So in brief the proper S6K, PLC $\gamma 1$, and BTK necessary for $\mathrm{PLC} \gamma 2$ productions which is necessary for B-cell maturation and T-cells modulations, and necessary for regulating thromboxane-A synthesis. Chronic lymphocytic leukemia [CLL] reflect Inhibition in BTK and in PLC $\gamma 2$ synthesis which reflect Inhibition or impaire in Thromboxane-A :

Proline amino acids are required for Collagen synthesis [32] where, Collagen binds to its receptors and activate both the PLC $\gamma 2-D A G-P K C$ and PI3 kinase/Akt-p38 MAPK cascades, where p38 MAPK can activate cPLA2, which catalyzes arachidonic acid (AA) release to produce thromboxane A2 (TxA 2 ) formation [33]

Bruton's tyrosine kinase "BTK" activates PLC $\gamma 2$ variants mediating ibrutinib resistance in human CLL . [34]

BTK inhibitors [ibrutinib , CNX-774] significantly attenuated TPA-induced cell invasion and migration in MCF-7 cells and inhibit the activation of the phospholipase $\mathrm{C} \gamma 2 / \mathrm{PKC} \beta$ signaling pathways [35]

BTK was initially shown to be defective in the primary immunodeficiency X-linked a gamma-globulinemia (XLA) and is essential both for B cell development and function of mature. [36] So, both of Collagen synthesis and BTK are the main functions for re-activating PLC $\gamma 2$ which catalyzes arachidonic acid (AA) release to produce thromboxane-A2 (TXA 2 ) formation (note the inhibition or mutation in BTK and PLC $\gamma 2$ will inhibit TXA2 synthesis and will cause Chronic lymphocytic leukemia ), where both BTK and PLC $\gamma 2$ are so necessary for B cells maturation and are critical for B-cell receptor (BCR), where, inhibition or reduction in BTK and in PLC $\gamma 2$ will reflect Inhibition in B-cells maturation, inhibition in T-cells modulations, and inhibitions in TXA2 synthesis and will be the result of Chronic lymphocytic leukemia "CLL" disease.

Vascular endothelial growth factor receptor (VEGFR) but not KIT, platelet-derived growth factor receptor (PDGFR) and FMSlike tyrosine kinase 3 (FLT3) are critical for CLL cell viability.

[37]

MTOR Ser Thr phosphorylation pathway regulate S6K production and promote VEGF activities for reproducing TXA2 (but through PLC $\gamma 2$ regulations) in one pathway, and the other pathway is stimulating the PLC $\gamma 1$ pruductions and promoting BTK activities for activating PLC $\gamma 2$ productions which will reactivate the proper TXA2 synthesis and mediate the activities of VEGF for producing TXA2, for reactivating tropomycine, 
and reactivating $\mathrm{G}$-actin filaments activities.

My note is, the synthesis of proper TXA2 in vivo are fully depending on PLC $\gamma 2$ and consequently on S6K and BTK activities and functions, but only VEGF are not enough and not satisfied for TXA2 synthesis. The proper S6K synthesis which will reactivate the PLC $\gamma 1$ and DTK which will promote the PLC 2 synthesis which I can consider it as the main necessary proper tools for TXA2 synthesis for blood synthesis, for bones maturations and for cells growth and then CLL cell viability.

So, PLC $\gamma 2$ (which basically regulated by ribosomes, by S6K, and by PLC $\gamma 1)$ promote TXA2 synthesis which can stimulate and reactivate VEGF synthesis upon feedback for tropomycine and for G-actin filaments reactivations for running full cellular Biosynthesis, for blood filtering in veins, and for cellular metabolism.

Chronic lymphocytic leukaemia (CLL) is a malignancy of CD5+ B cells that is characterized by the accumulation of small, mature-appearing lymphocytes in the blood, in bone marrow and in lymphoid tissues due to PLC $\gamma 2$ inhibition may due to full mutated S6K production .

PLC $\gamma 2$ synthesis occurred mainly in bone marrow where normal blood synthesis is regulated by skeletal tissue that is having orders from basic ribosomes ,but mature CLL blood are activated and formed only by the activities of mTOR Ser/ Thr signaling which promote the VEGF, toropomycine synthesis (where both cannot promote TXA2 synthesis without PLC $\gamma 2$ availability ) that both VEGF and toropomycine are necessary for reactivate G-actin filaments and re purify blood in veins.

So why VEGF +toropomycine is producing white mature cells?? VEGF can not regulate directly the PLC $\gamma 2$ synthesis and consequently can't regulate TXA2 synthesis but TXA2 synthesis can not be done without PLC $\gamma 2$ regulations .

Where VEGF responsible for increasing the plasma long livedplasma cells (LLPC), then the generation of antigen-specific antibody for Durable humoral immunity (which produced by non-proliferating bone marrow. [38]

Old blood cells when passes through spleen will be broken to save iron which bind to PLC $\gamma 2$ for regenerate new blood cells by PLC $\gamma 2$ which extracted in spleen which are responsible for metals transportations and proliferation for new cells, but inhibition in PLC $\gamma 2$ with increasing in the mutated S6K will inhibit TXA2 synthesis and will increase long lived plasma which increased by increasing in nutrients-mTOR signalling. The B cell receptor (BCR) signaling pathway (which regulated by PLC $\gamma 2$ synthesis and activities) has critical cell survival implications in B-cells malignancies, such as chronic lymphocytic leukemia (CLL). small molecule tyrosine kinase inhibitors of members of the BCR signaling pathway have proven to be transformational in treatment of CLL. [39] The B-cell receptor (BCR) is a key survival molecule for normal B cells and for most B-cell malignancies.

In CLL, engagement of the BCR (which regulated by PLC $\gamma 2$ ) by antigen occurs in vivo, leading to down-regulated expression and to an unanticipated modulation of glycosylation of surface $\operatorname{IgM} \cdot[40]$

So inhibition in PLC $\gamma 2$ synthesis will inhibit BCR signalling function that will lead to inhibition in modulation in $\operatorname{IgM}$ which normally done by BCR function for activating B-cells maturation.

$\operatorname{IgM}$ autoantibodies, and the evidence that these anti-apoptotic cell IgM natural antibodies can regulate inflammatory responses through ancient pathways of the innate immune system that first arose long before the initial emergence of the adaptive immune system.

My note,

PLC $\gamma 2$ first regulate BCR activities which regulate both IgM and $\operatorname{IgD}$ synthesis through synthase enzyme regulation, where $\operatorname{IgM}$ is more active and less stable than $\operatorname{IgD}$ that $\operatorname{IgM}$ necessary for modulating and regulating inflammatory immune response and antiinflammatory processes through modulating T-cells reactivity.

\section{Results and Conclusion :}

Chronic lymphocytic leukemia [CLL] reflect Inhibition in PLC $\gamma 2$ synthesis "may due to inhibition in OPA1 synthase" lead to inhibition in CXCR12 where CXCR12 is the main activator and regulator for CXCR4 synthesis Upton phospholipase effects on CXCR12.

Also inhibition in PLC $\gamma 2$ Bio-Synthesis will reflect reduction or inhibition in thromboxane-A production.

Osteoarthritis "OA" is characterized by a sharp expression in Gamma-Phospholipase C-1 "PLC $\gamma 1$ " (which catabolize inflammations), with decreasing "or inhibition" in PLC $\gamma 2$ "PLC beta" productions (which necessary for immune modulation, for B-cell maturation and for T-cells modulation and regulate TXA2 synthesis).

The increasing in PLC $\gamma 1$ with Deficiency in Ser amino acids, and deficiency in proper S6K, with decreasing or inhibition in OPA1-synthase activity will lead to inhibition in PLC $\gamma 2$ which lead to diabetes and early Osteoarthritis"OA" prognosis .

PLC $\gamma 2$ are so necessary for re-modulating T-cells and immune efficiencies, and necessary for regulating antigen and thromboxane-A synthesis.

The inhibitions or reduction or mutations in BTK and in its main proper PLC $\gamma 2$ producions will cause an inherent inhibition or reduction in CXCL12 then will be followed by inhibition or reduction in CXCR4 then will lead to inhibition in the regulation of B-cell maturation, migration, adhesion, and also lead to severe decreasiing in anti-inflammatory processes of immune productive efficiency.

Also inhibition in BTK and PLC $\gamma 2$ mainly will reflect Inhibition in the two antigens $\operatorname{IgM}$ in and IgD synthesis.

Chronic lymphocytic leukemia "CLL" reflect decreasing or inhibition on growth-promoting signaling via the B-cell receptor. The Bruton tyrosine kinase (BTK) is the important for PLC $\gamma 2$ systems which is necessary for B-cell activities and T-cells modulation.

Bruton tyrosine kinase (Btk) necessary to activates PLC $\gamma 2,11,12$ which necessary to activate thromboxane A2 and necessary for modulating immune activities and T-cells too.

Both Collagen and BTK pathways are necessary tools for reactivating PLC $\gamma 2$ which catalyzes arachidonic acid (AA) release to produce thromboxane-A2 (TXA 2 ) synthesis, and necessary for $\mathrm{B}$ cells maturation and critical for B-cell receptor (BCR), where, inhibition in BTK and in PLC $\gamma 2$ will reflect diabetes, Osteoarthritis, and the Chronic lymphocytic leukemia "CLL" disease depending on the percentage of Ser \& hydroponic amino acids shortage and depending on the percentage of inhibition of necessary pathways needed for PLC $\gamma 2$ synthesis and reactivities. Also, inhibition in the availability of Ser, Tyr, Leu , Pro with inhibition in necessary hydrophobic amino acids synthesis and in BTK and then in PLC $\gamma 2$ can lead to Osteosarcoma which is a cancer cases that produces immature bone (due to mutins in PLC $\gamma 2$ and in TLR4 productions) found at the end of long bones, often around the knee.

Deficiency in proline with inhibition in Ser, Tyr, leu (or mutations 
in synthase) and in specific beta-subunits-calcium carriar can reflect mutations in the PLC $\gamma 2$ (beta subunits) productions due to deficiency in proper beta-oxidation that can lead to deficiency or inhibition in the PLC $\gamma 2$ and PLC alpha, and in MHC class two, that will lead to deficiency or inhibition "or mutations" in "SIRP $\alpha 1$ and in TLR4, PD-L1 then in PD-L1" lead to isolations to that area (due to precipitation of the un functioned calcium by PLCs) that can lead to mutated immature bone and tissue synthesis.

\section{Conflict Of Interest Statement :}

The Author declare that the research work has been conducted in the absence of any commercial or financial relationships, that could be construed as a potential conflict of interest.

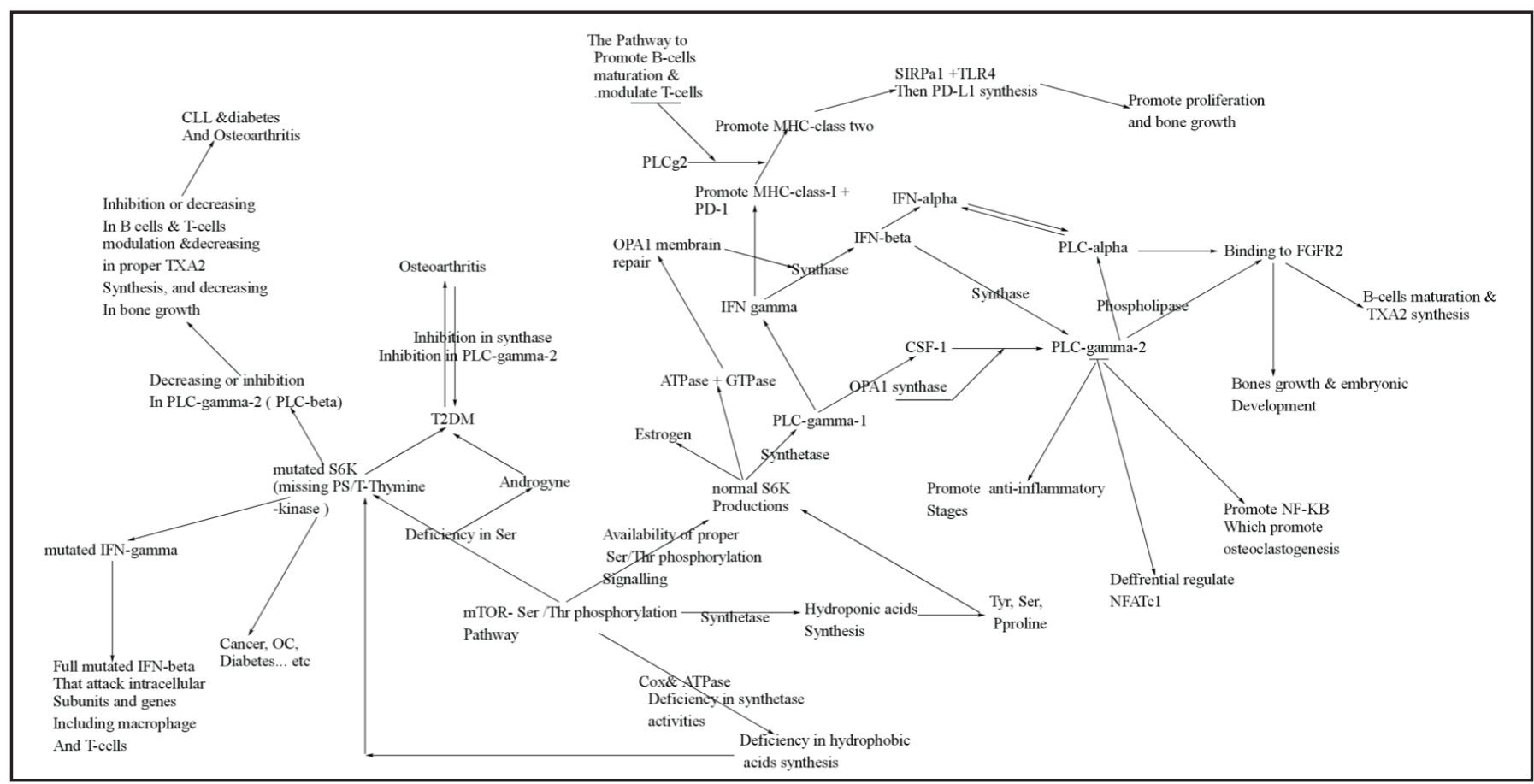

Figure 1 - Osteoarthritis linked with diabetes BTK and PLCg2 regulate thromboxane-A Synthesis where their inhibition or mutation reflect CLL diseases. _discrimination of PLCg2 pathway for modulating T-cells, B-cells maturation and bones growth.

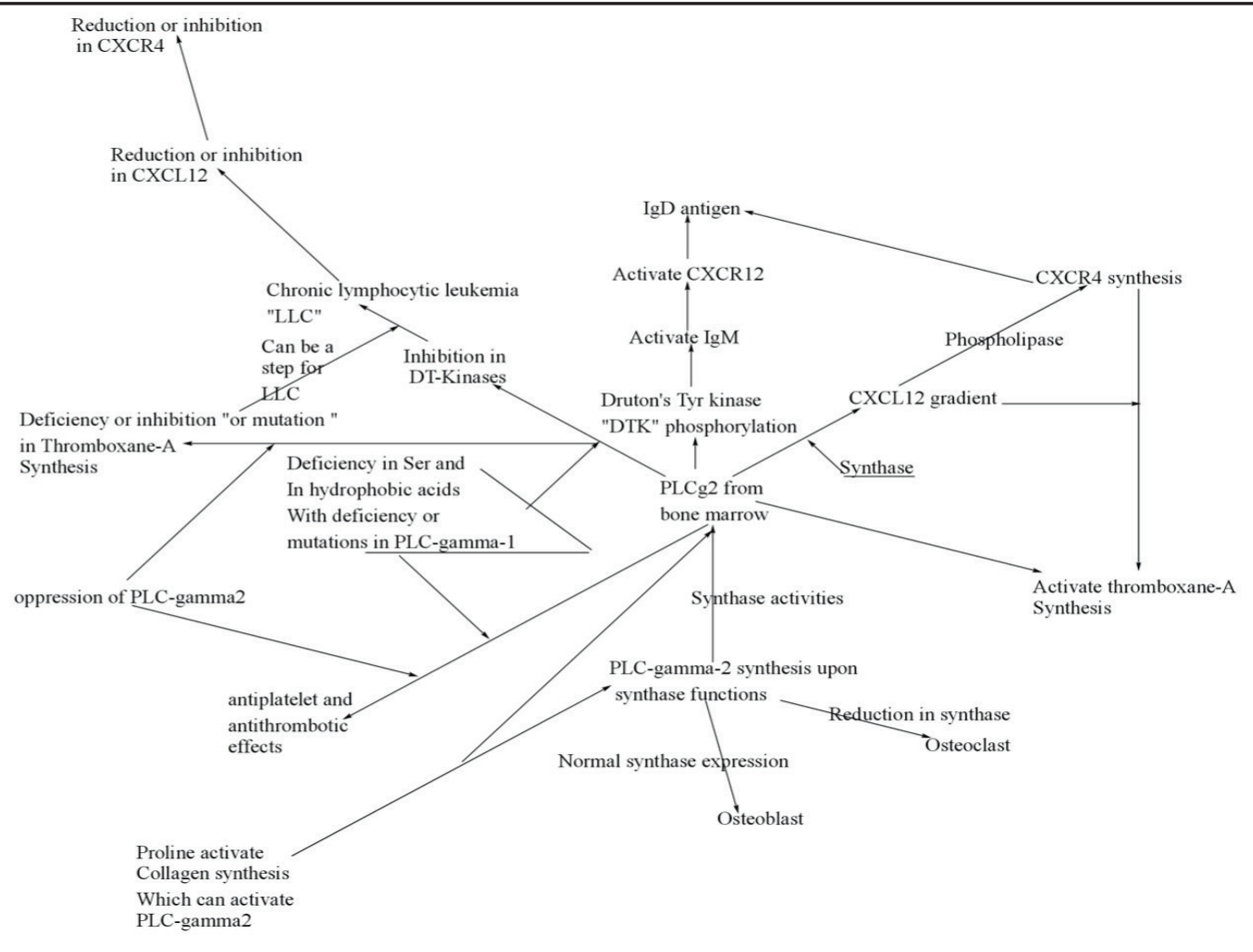

Figure 2 - PLC-gamma-2 regulate both CXCL 12 and CXCR4 upondruton's tyrosine Kinases “DTK” phosphorylation. 


\section{References:}

1. Disruption of Phosphoinositide-Specific Phospholipases C $\gamma 1$ Contributes to Extracellular Matrix Synthesis of Human Osteoarthritis Chondrocytes,August 2014, International Journal of Molecular Sciences 15(8):13236-46 DOI:10.3390/ ijms150813236 PubMed.

2. PLC $\gamma 1$ inhibition-driven autophagy of IL-1 $\beta$-treated chondrocyte confers cartilage protection against osteoarthritis, involving AMPK, Erk and Akt Xiaolei Chen, Yue Wang,Ning Qu,Bing Zhang,Chun Xia First published: 28 December 2020 https://doi.org/10.1111/jcmm.16245

3. Ashraf M El T. An In-Depth Study of TCA cycles, OPA1, S6K1, ATPase, TLR4, MHC-class-I, GCs, and IFNs Biosynthesis, and Their Roles of Deficiency in Diabetes, Asthma, Cancer, etc. and the NAD Roles in their Activities. 2021- 4(6)OAJBS.ID.000331. DOI: $10.38125 / \mathrm{OAJBS} .000331$

4. Yu, M., Chen, Y., Zeng, H. et al. PLC $\gamma$-dependent mTOR signalling controls IL-7-mediated early B cell development. Nat Commun 8, 1457 (2017). https://doi.org/10.1038/s41467-01701388-5

5. Citation: Ashraf Marzouk El Tantawi (2021) An In-Depth Study of TCA cycles, OPA1, S6K1, ATPase, TLR4, MHC-class-I, GCs, and IFNs Bio-Synthesis, and their Roles of Defciency in Diabetes, Asthma, Cancer, etc and the NAD Roles in their Activities. Japan Journal of Clinical \& Medical Research. SRC/ JJCMR-118. DOI: doi.org/10.47363/JJCMR/2021(1)115Jap

6A*, Ashraf M El T. An In-Depth Study of TCA cycles, OPA1, S6K1, ATPase, TLR4, MHC-class-I, GCs, and IFNs Biosynthesis, and Their Roles of Deficiency in Diabetes, Asthma, Cancer, etc. and the NAD Roles in their Activities. 2021- 4(6) OAJBS.ID.000331. DOI: 10.38125/OAJBS.000331

6. The role of PLC $\gamma 2$ in immunological disorders,, cancer, and neurodegeneration Received for publication, October 13, 2020, and in revised form, June 15, 2021 Published, Papers in Press, June 19, 2021, https://doi.org/10.1016/j.jbc.2021.100905 Jacob T. Jackson, Elisabeth Mulazzani, Stephen L. Nutt,, and Seth L. Masters

7. Type 2 diabetes mellitus and osteoarthritis Author links open overlay panelNicolaVeroneseaAndréScheent

https://doi.org/10.1016/j.semarthrit.2019.01.005

8. Insulin Activates ATP-Sensitive $\mathrm{K}+$ Channels in Pancreatic $\beta$-Cells Through a Phosphatidylinositol 3-Kinase-Dependent Pathway Farrukh A. Khan, Paulette B. Goforth, Min Zhang and Leslie S. Satin +Author Affiliations Diabetes 2001 Oct; 50(10): 2192-2198. https://doi.org/10.2337/diabetes.50.10.2192

9. The roles of protein tyrosine phosphatases in bone-resorbing osteoclasts Authorlinks open overlay panelMoranShalevAriElson https://doi.org/10.1016/j.bbamcr.2018.07.005 Molecular Cell Research Volume 1866, Issue 1, January 2019, Pages 114-123.

10. Tyrosine phosphatases as regulators of skeletal development and metabolism Katherine R. Schiller,Laura J. Mauro published: 28 July 2005 https://doi.org/10.1002/jcb.20515
11. Buckbinder L, Crawford DT, Qi H, Ke HZ, Olson LM, Long KR, Bonnette PC, Baumann AP, Hambor JE, Grasser WA 3rd, Pan LC, Owen TA, Luzzio MJ, Hulford CA, Gebhard DF, Paralkar VM, Simmons HA, Kath JC, Roberts WG, Smock SL, Guzman-Perez A, Brown TA, Li M. Proline-rich tyrosine kinase 2 regulates osteoprogenitor cells and bone formation, and offers an anabolic treatment approach for osteoporosis. Proc Natl Acad Sci U S A. 2007 Jun 19;104(25):10619-24. doi: 10.1073/ pnas.0701421104. Epub 2007 May 30. PMID: 17537919; PMCID: PMC1880863.

12. Timsah Z, Ahmed Z, Lin CC, Melo FA, Stagg LJ, Leonard PG, Jeyabal P, Berrout J, O’Neil RG, Bogdanov M, Ladbury JE. Competition between Grb2 and Plc $\gamma 1$ for FGFR2 regulates basal phospholipase activity and invasion. Nat Struct Mol Biol. 2014 Feb;21(2):180-8. doi: 10.1038/nsmb.2752. Epub 2014 Jan 19. PMID: 24440983.

13. Zhu L, Jones C, Zhang G. The Role of Phospholipase $\mathrm{C}$ Signaling in Macrophage-Mediated Inflammatory Response. J Immunol Res. 2018 Feb 8;2018:5201759. doi: 10.1155/2018/5201759. PMID: 30057916; PMCID: PMC6051040.

14. Cellular Signalling Volume 73, September 2020, 109673 The adaptor protein APS modulates BCR signalling in mature B cells Author links open overlay panelElisabettaDondiabLauraVelazquezab1 https://doi.org/10.1016/j.cellsig.2020.109673

15. Phospholipase $\mathrm{C} \gamma 1$ (PLC $\gamma 1$ ) Controls Osteoclast Numbers via Colony-stimulating Factor 1 (CSF-1)-dependent Diacylglycerol/ $\beta$-Catenin/CyclinD1 Pathway* Zhengfeng Yang, Seokho Kim, [...], and Roberta Faccio Yang, Seokho Kim, [...], and Roberta Faccio Article information J Biol Chem. 2017 Jan 27; 292(4): 1178-1186. Published online 2016 Dec 9. doi: 10.1074/jbc. M116.764928 PMCID: PMC5270464 PMID: 27941021

16. Mun, S.H., Park, P.S.U. \& Park-Min, KH. The M-CSF receptor in osteoclasts and beyond. Exp Mol Med 52, 1239 1254 (2020). https://doi.org/10.1038/s12276-020-0484-z

17. Gao B, Xu W, Wang Y, Zhong L, Xiong S. Induction of TRIM22 by IFN- $\gamma$ Involves JAK and PC-PLC/PKC, but Not MAPKs and $\mathrm{pI} 3 \mathrm{~K} / \mathrm{Akt} / \mathrm{mTOR}$ Pathways. J Interferon Cytokine Res. 2013 Oct;33(10):578-87. doi: 10.1089/jir.2012.0170. Epub 2013 May 9. PMID: 23659673; PMCID: PMC3793658.

18. Interferon- $\gamma$-induced Epithelial ICAM-1 Expression and Monocyte Adhesion Involvement Of Protein Kinase C-Dependent C-Src Tyrosine Kinase Activation Pathway* YaJen Chang Michael J. Holtzman Ching-Chow Chen Access DOI:https://doi.org/10.1074/jbc.M109924200

19. Lee, AM., States, D. Colony-stimulating factor-1 requires PI3-kinase-mediated metabolism for proliferation and survival in myeloid cells. Cell Death Differ 13, 1900-1914 (2006). https://doi.org/10.1038/sj.cdd.4401884

20. Wang, H., Altomare, D., Skele, K. et al. Positive feedback regulation between AKT activation and fatty acid synthase expression in ovarian carcinoma cells.

Oncogene 24, 3574-3582 (2005). https://doi.org/10.1038/ sj.onc. 1208463 
21. Phospholipase C $\gamma 1$ (PLC $\gamma 1$ ) Controls Osteoclast Numbers via Colony-stimulating Factor 1 (CSF-1)-dependent Diacylglycerol/ $\beta$-Catenin/CyclinD1 Pathway* Zhengfeng Yang, Seokho Kim, [...], and Roberta Faccio J Biol Chem. 2017 Jan 27; 292(4): 1178-1186. Published online 2016 Dec 9. doi: 10.1074/jbc. M116.764928 PMCID: PMC5270464 PMID: 27941021

22. PLC $\gamma 2$ regulates osteoclastogenesis via its interaction with ITAM proteins and GAB2 Dailing Mao, Holly Epple, [...], and Roberta Faccio J Clin Invest. 2006 Nov 1; 116(11): 2869-2879. Published online 2006 Oct 19. doi: 10.1172/JCI28775 PMCID: PMC1616195 PMID: 17053833

23. Phospholipase C $\gamma 2$ Is Critical for Dectin-1-mediated Ca2+ Flux and Cytokine Production in Dendritic Cells* Shengli Xu, Jianxin Huo, [...], and Kong-Peng Lam J Biol Chem. 2009 Mar 13; 284(11): 7038-7046.Doi: 10.1074/jbc.M806650200 PMCID: PMC2652331 PMID: 19136564

24. Phospholipase C Gamma 2 Is Critical for Development of a Murine Model of Inflammatory Arthritis by Affecting Actin Dynamics in Dendritic Cells Viviana Cremasco, Elisa Benasciutti, [...], and Roberta Faccio PLoS One. 2010; 5(1): e8909. Published online 2010 Jan 27. doi: 10.1371/journal. pone.0008909 PMCID: PMC2811739 PMID: 20111715

25. Phospholipase $\mathrm{C} \gamma 2$ plays a role in T-cell receptor signal transduction and T-cell selection1 Guoping Fu, Yuhong Chen, [...], and Renren Wen J Immunol. 2012 Sep 1; 189(5): 2326-2332. Published online 2012 Jul 25. doi: 10.4049/jimmunol.1103458 PMCID: PMC3528184

26. Properdin and factor $\mathrm{H}$ production by human dendritic cells modulates their $\mathrm{T}$ cell stimulatory capacity and is regulated by IFN $\gamma$ Karen O. Dixon, Joseph O'Flynn, [...], and Cees van Kooten. Eur J Immunol. 2017 Mar; 47(3): 470-480.

Published online 2017 Mar 13. doi: 10.1002/eji.201646703 PMCID: PMC5363362.

27. The role of PLC $\gamma 2$ in immunological disorders, cancer, and neurodegeneration Author links open overlay panelJacob T.Jackson12 Seth L.Masters234 https://doi.org/10.1016/j. jbc.2021.100905

28. Biochem J. 1980 Jan 1; 185(1): 1-11. doi: 10.1042/bj1850001 PMCID: PMC1161263 PMID: 7378042 Fibrinogen-fibrin conversion. The mechanism of fibrin-polymer formation in solution Gerald F. Smith

29. Signaling During Platelet Adhesion and Activation Zhenyu Li, M. Keegan Delaney, Kelly A. O’Brien, and Xiaoping Du Originally published11 Nov 2010https:// doi.org/10.1161/ATVBAHA.110.207522Arteriosclerosis, Thrombosis, and Vascular Biology. 2010;30:2341-2349

30. Front. Pharmacol., 06 November 2018 | https://doi. org/10.3389/fphar.2018.01293 Phospholipase C $\gamma 2$ Signaling Cascade Contribute to the Antiplatelet Effect of Notoginsenoside Fc Yingqiu Liu1, Tianyi Liu2, Kevin Ding3, Zengyuan Liu1,[...] Li Cui4 and Xiaoping Song1

31. [Activation of Platelet Function Through G ProteinCoupled Receptors Stefan Offermanns Originally published8 Dec 2006https://doi.org/10.1161/01.
RES.0000251742.71301.16Circulation

Research. 2006;99:1293-1304].

32. Front. Oncol., 27 August 2021 | https://doi.org/10.3389/ fonc.2021.719922 Regulation of Extracellular Matrix Production in Activated Fibroblasts: Roles of Amino Acid Metabolism in Collagen Synthesis Emily J. Kay1*, Grigorios Koulouras1,2 and Sara Zanivan1,2

33. Lu, WJ., Lee, JJ., Chou, DS. et al. A novel role of andrographolide, an NF-kappa B inhibitor, on inhibition of platelet activation: the pivotal mechanisms of endothelial nitric oxide synthase/cyclic GMP. J Mol Med 89, 1261-1273 (2011). https://doi.org/10.1007/s00109-011-0800-0 ]]]]]

34. Wist M, Meier L, Gutman O, Haas J, Endres S, Zhou Y, Rösler R, Wiese S, Stilgenbauer S, Hobeika E, Henis YI, Gierschik P, Walliser C. Noncatalytic Bruton's tyrosine kinase activates PLC $\gamma 2$ variants mediating ibrutinib resistance in human chronic lymphocytic leukemia cells. J Biol Chem. 2020 Apr 24;295(17):5717-5736. doi: 10.1074/jbc.RA119.011946. Epub 2020 Mar 17. PMID: 32184360; PMCID: PMC7186163.

35. Bruton's agammaglobulinemia tyrosine kinase (Btk) regulates TPA-induced breast cancer cell invasion via PLC $\gamma 2 /$ PKC $\beta / \mathrm{NF}-\kappa \mathrm{B} / \mathrm{AP}-1-d e p e n d e n t$ matrix metalloproteinase-9 activation Authors: Jeong-Mi Kim Jinny Park Eun-Mi Noh Hyun-Kyung Song Sang Yull Kang Sung Hoo Jung Jong-Suk Kim Byung-Hyun Park Young-Rae Lee Hyun Jo Youn View Affiliations Published online on: March 9, 2021 https:// doi.org/10.3892/or.2021.8007

36. Role of Bruton's tyrosine kinase in B cells and malignancies Pal Singh, S., Dammeijer, F. \& Hendriks, R.W. Role of Bruton's tyrosine kinase in B cells and malignancies. Mol Cancer 17, 57 (2018). https://doi.org/10.1186/s12943-018-0779-z

37. Fecteau, JF., Bharati, I.S., O’Hayre, M. et al. SorafenibInduced Apoptosis of Chronic Lymphocytic Leukemia Cells Is Associated with Downregulation of RAF and Myeloid Cell Leukemia Sequence 1 (Mcl-1). Mol Med 18, 19-28 (2012). https://doi.org/10.2119/molmed.2011.00164

38. Survival of Long-Lived Plasma Cells (LLPC): Piecing Together the Puzzle Shivana M. Lightman, Adam Utley and Kelvin P. Lee Front. Immunol., 03 May 2019 | https://doi. org/10.3389/fimmu.2019.00965

39. The Role of BTK Inhibition in the Treatment of Chronic Lymphocytic Leukemia: A Clinical View Authors Tambaro FP, De Novellis D, Wierda WG Published 29 October 2021 Volume 2021:13 DOI https://doi.org/10.2147/JEP.S265284

40. B-cell receptor signaling in chronic lymphocytic leukemia Freda K. Stevenson, Sergey Krysov, Andrew J. Davies, Andrew J. Steele, Graham Packham Blood (2011) 118 (16): 4313-4320. https://doi.org/10.1182/blood-2011-06-338855

41. Natural IgM: Beneficial autoantibodies for the control of inflammatory and autoimmune disease?

Caroline Grönwall and Gregg J. Silverman

ClinImmunol. Author manuscript; available in PMC $2015 \mathrm{Jul} 1$. Published online 2014 Apr 2. doi: 10.1007/s10875-014-0025-4 PMCID: PMC4354681 